
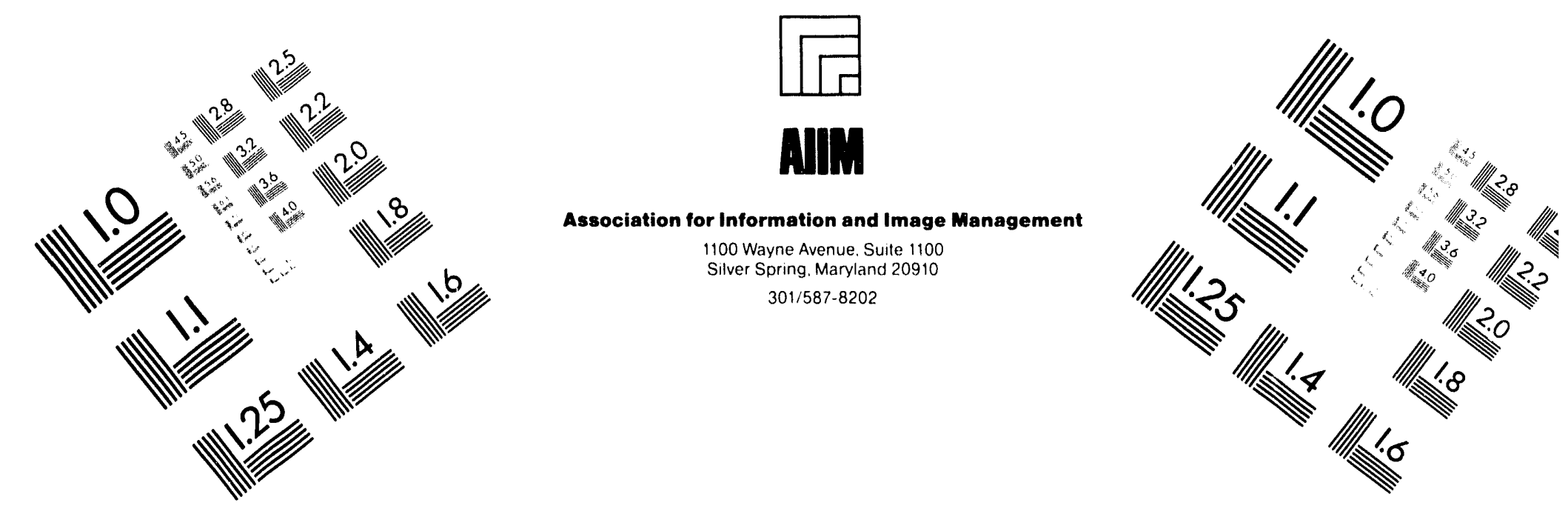

\title{
Centimeter
}

10

Inches
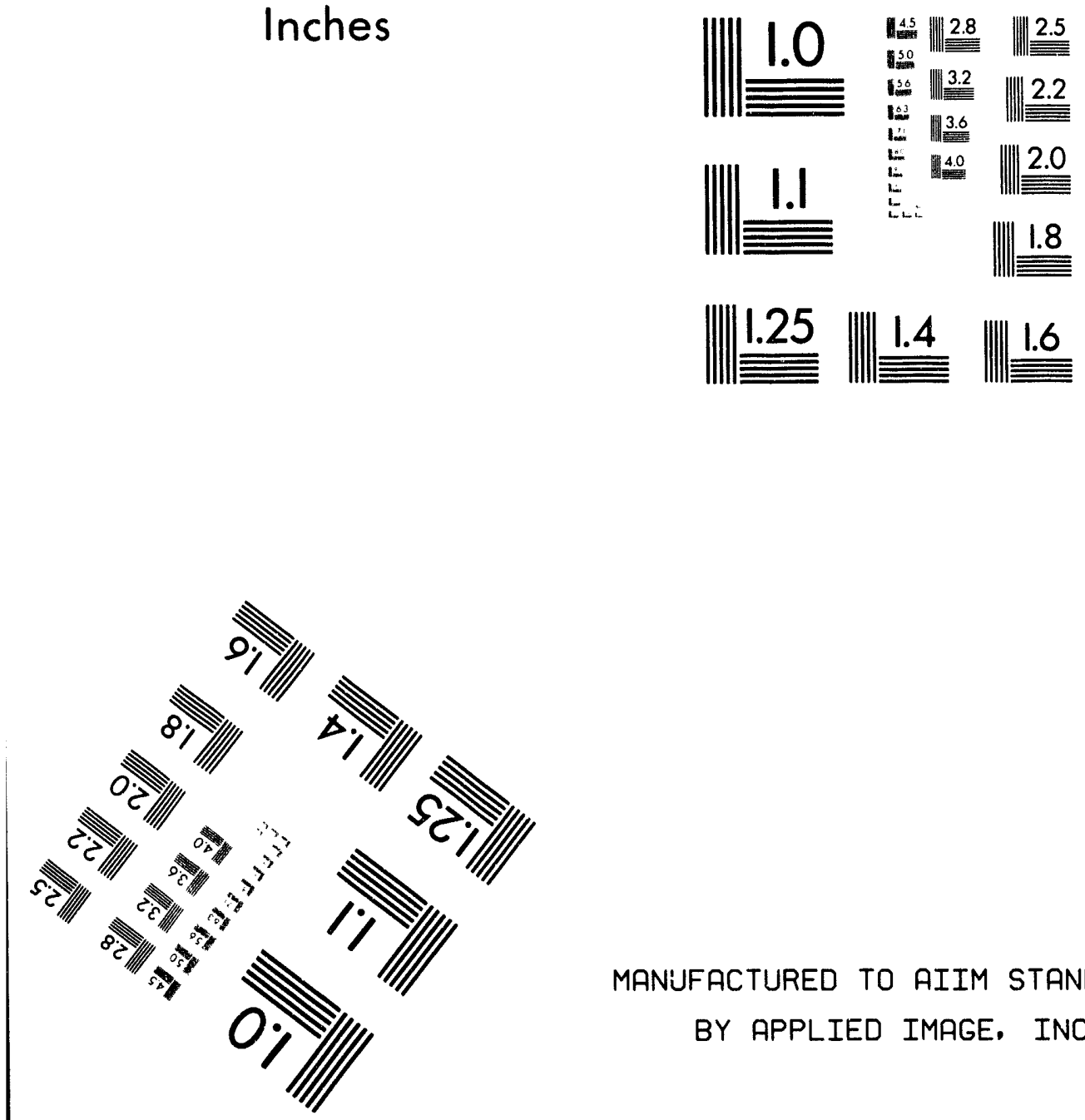

MANUFACTURED TO AIIM STANDARDS

BY APPLIED IMAGE. INC.

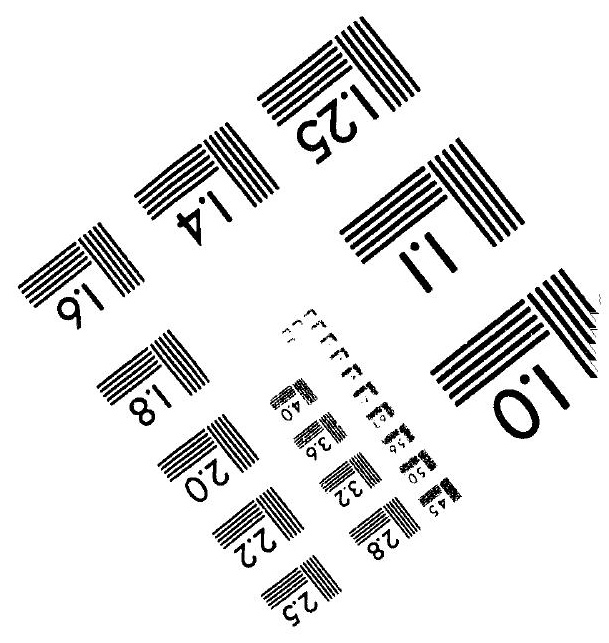



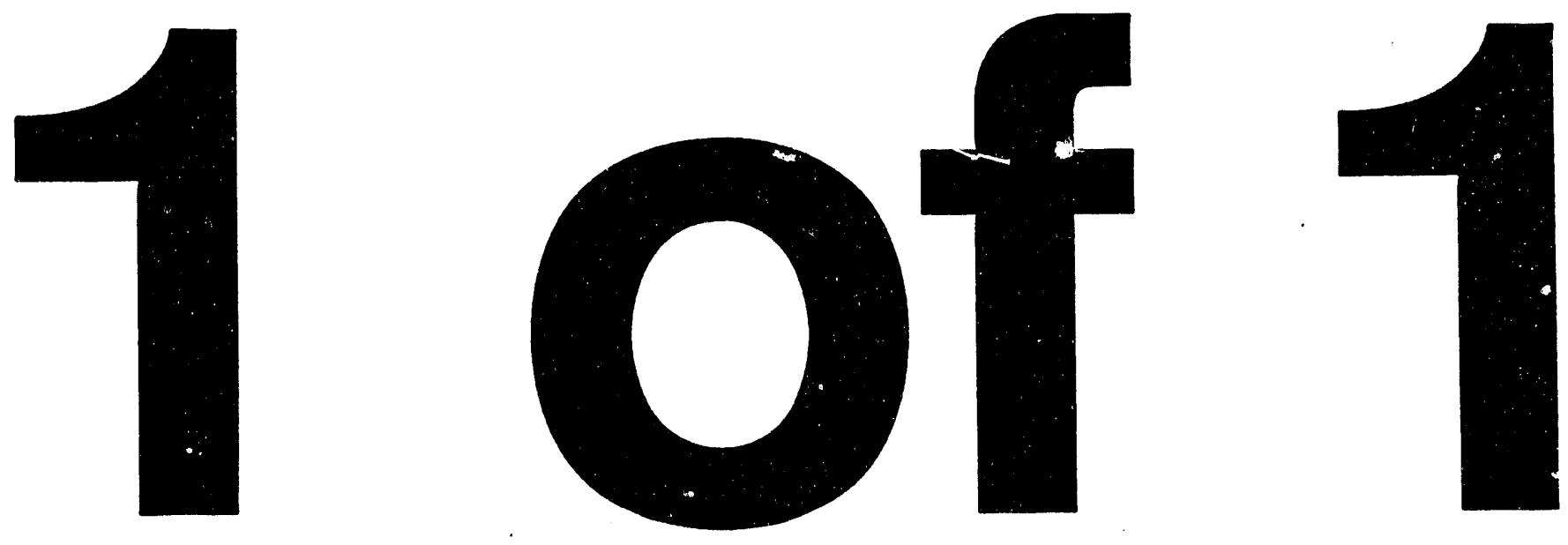


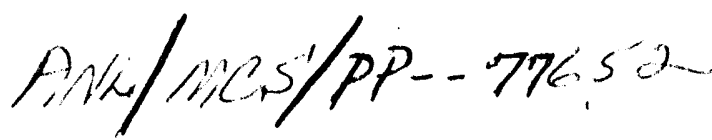

\section{Asymptotics of a Free Boundary Problem*}

\author{
F. V. Atkinson`, Hans G. Kaper, Man Kam Kwong \\ Mathematics and Computer Science Division \\ Argonne National Laboratory \\ Argonne, IL 60439-4801
}

October 5, 1992

\begin{abstract}
This article is concerned with free boundary problems for the differential equation

$$
u^{\prime \prime}+\frac{2 \nu+1}{r} u^{\prime}+u-u^{q}=0, r>0
$$

where $0 \leq q<1$ and $\nu \geq 0$. As was shown by Kaper and Kwong [Differential and Integral Equations 3, 353-362], there exists a unique $R>0$, such that the equation admits a classical solution $u$ that is positive and monotone on $(0, R)$ and that satisfies the boundary conditions

$$
u^{\prime}(0)=0, u(R)=u^{\prime}(R)=0 .
$$
\end{abstract}

This article is concerned with the behavior of $R$ and $u(0)$ as $q \rightarrow 1$.

AMS(MOS) Subject Classification. Primary 34B15. Secondary 35J25, $35 \mathrm{~J} 65$.

Key Words and Phrases. Reaction-diffusion equation, free boundary problem, radial solution, Emden-Fowler equation, positive solution, asymptotic behavior.

\section{The Problem}

We consider free boundary problems for the reaction-diffusion equation $\Delta u+u-u^{q}=0$ $(0 \leq q<1)$ in $\mathbf{R}^{N}(N=2,3, \ldots)$. In particular, we are interested in radial solutions-that

- This work was supported by the Applied Mathematical Sciences subprogram of the Office of Energy Research, U. S. Department of Energy, under Contract W-31-109-Eng-38.

'Permanent address: Department of Mathematics, University of Toronto, Toronto, M5S 1A1, Ontario, Cianada. 
is, solutions that depend only on the radial variable $r=|x|$. Such solutions satisfy the differential equation

$$
u^{\prime \prime}+\frac{2 \nu+1}{r} u^{\prime}+u-u^{q}=0, r>0,
$$

where $\nu=\frac{1}{2}(N-2)$. Without loss of generality we will consider $\nu$ as a real number, not necessarily half-integer $(\nu \geq 0)$.

As was shown by Kaper and Kwong [1], there exists, for each $q \in[0,1)$, a unique $R>0$, such that (1.1) admits a classical solution $u$ that is positive and monotone on $(0, R)$ and that satisfies the boundary conditions

$$
u^{\prime}(0)=0, u(R)=u^{\prime}(R)=0 .
$$

In this article, we investigate the behavior of $R$ and $u(0)$ as $q \rightarrow 1$.

\section{The Result}

We prove the following result.

Theorem 1 For each $q \in[0,1)$, there is a unique $R>0$ such that (1.1),(1.2) admits a (classical) solution $u$ that is positive everywhere on $(0, R)$. The function $u$ is monotonically decreasing on $(0, R) ;\|u\|$ is bounded, but $R$ grows beyond bounds as $q \rightarrow 1$.

In the special case $\nu=\frac{1}{2}(N=3)$ we have a lower bound on $R$,

$$
R>\sqrt{\frac{2}{1-q}}, \quad 0 \leq q<1,
$$

but, as we do not have a comparable upper bound, we cannot conclude that $R=\mathrm{O}((1-$ $q)^{-1 / 2}$ ) as $q-1$.

\section{The Proof}

Using a shooting argument, we replace the boundary value problem (1.1), (1.2) by the initial value problem

$$
\begin{gathered}
u^{\prime \prime}+\frac{2 \nu+1}{r} u^{\prime}+u-u^{q}=0, r>0, \\
u(0)=\gamma, u^{\prime}(0)=0 .
\end{gathered}
$$

The results of [1] imply that, for any $q \in[0,1)$, there is a unique $\gamma>1$, such that the solution of (3.1), (3.2) decreases from $\gamma$ to meet the $r$-axis with zero slope at some value $R>0$. Denoting this solution by $u(\cdot, \gamma)$, we have

$$
u(R, \gamma)=0, u^{\prime}(R, \gamma)=0 .
$$

The lower bound on $\gamma$ can be sharpened to $(2 /(1+q))^{1 /(1-q)}$, but 1 suffices for our purpose. The proof consists of a detailed investigation of the behavior of $u(\cdot, \gamma)$. 


\subsection{Down to 1 .}

We begin 1 y showing that $u(r, \gamma)$ decreases monotonically from the value $\gamma$ at $r=0$ to the value 1 at some finite point $r_{0}$.

Lemma 1 There exists a point $r_{0}<j_{\nu, 1} /(1-q)^{1 / 2}$, such that $u(\cdot, \gamma)$ is monotonically decreasing on $\left(0, r_{0}\right)$, with $u\left(r_{0}, \gamma\right)=1$ and $u^{\prime}\left(r_{0}, \gamma\right)<0$. Here, $j_{\nu, 1}$ is the first positive zero of $J_{\nu}$-the Bessel function of the first kind of order $\nu$.

Proof. As long as $u>1$, we have $u-u^{q}>(1-q) u$, so $u(\cdot, \gamma)$ oscillates faster than the solution $v$ of the equation

$$
v^{\prime \prime}+\frac{2 \nu+1}{r} v^{\prime}+(1-q) v=0 \text {. }
$$

In particular, $u(\cdot, \gamma)$ reaches the value 1 before $v$ does. Now, $v(r)$ is a constant multiple of $r^{-\nu} J_{\nu}\left(r(1-q)^{1 / 2}\right)$, where $J_{\nu}$ is the Bessel function of the first kind of order $\nu$-see, for example, [2]. Hence, $v(r)=1$ for some value $r<j_{\nu, 1} /(1-q)^{1 / 2}$, where $j_{\nu, 1}$ is the first positive zero of $J_{\nu}$. We conclude that there must be a point $r_{0}<j_{\nu, 1} /(1-q)^{1 / 2}$, such that $\gamma>u(r, \gamma)>1$ for $0<r<r_{0}$ and $u\left(r_{0}, \gamma\right)=1$.

Since $u^{\prime}(0, \gamma)=0$ and $u^{\prime \prime}(r, \gamma)<0$ near 0 , it must be the case that $u^{\prime}(r, \gamma)<0$ near 0 .

Suppose $u(\cdot, \gamma)$ were not monotone on $\left(0, r_{0}\right)$. Then there exists a value $r_{1} \in\left(0, r_{0}\right)$ where $u(r, \gamma)$ has a local minimum, with $u\left(r_{1}, \gamma\right)>1$. Because $u(r, \gamma)$ reaches the value 1 at $r_{0}$, there must then exist a value $r_{2} \in\left(r_{1}, r_{0}\right)$ such that $u\left(r_{2}, \gamma\right)=u\left(r_{1}, \gamma\right)$ and $u^{\prime}\left(r_{2}, \gamma\right) \leq 0$. Multiplying the differential equation (3.1) by $u^{\prime}$ and integrating over $\left(r_{1}, r_{2}\right)$, we find that

$$
\frac{1}{2}\left(u^{\prime}\left(r_{2}, \gamma\right)\right)^{2}=-(2 \nu+1) \int_{r_{1}}^{r_{2}} \frac{\left(u^{\prime}(r, \gamma)\right)^{2}}{r} d r
$$

But here we have a contradiction, as the two sides of this identity have opposite signs. It must therefore be the case that $u(\cdot, \gamma)$ is monotone on $\left(0, r_{0}\right)$.

The monotonicity of $u(\cdot, \gamma)$ on $\left(0, r_{0}\right)$ implies that $u^{\prime}\left(r_{0}, \gamma\right) \leq 0$. If $u^{\prime}\left(r_{0}, \gamma\right)=0$, then it follows from the Lipschitz continuity of the function $u-u^{q}$ for $u>0$ and the consequential uniqueness of the solution of the intial value problem for (3.1) in the direction of decreasing $r$ starting at $r=r_{0}$ that $u(r, \gamma)=1$ for all $r \in\left(0, r_{0}\right)$. But then we have a contradiction, as $u(0, \gamma)=\gamma>1$. We conclude that $u^{\prime}\left(r_{0}, \gamma\right)<0$.

\subsection{Beyond $r_{0}$.}

From Lemma 1 we know that $u(r, \gamma)$ decreases monotonically until it reaches the value 1 with a negative slope at $r=r_{0}$. Beyond $r_{0}, u(r, \gamma)$ decreases further until it either reaches the value 0 with a negative or zero slope, or it bottoms out at some finite value of $r$ with a minimum value between 0 and 1 . 
Let $r_{1}$ be the point where $u(r, \gamma)$ ceases to be positive,

$$
r_{1}=\sup \left\{r>r_{0}: u(s, \gamma)>0,0<s<r\right\} \text {. }
$$

If $r_{1}$ is finite and $u\left(r_{1}, \gamma\right)=0$, we do not consider $u(\cdot, \gamma)$ beyond $r_{1}$. In this case, we can use the same argument as in the proof of Lemma 1 to show that $u(\cdot, \gamma)$ is monotonically decreasing on the entire interval $\left(0, r_{1}\right)$. In particular, if $\gamma$ is such that not only $u\left(r_{1}, \gamma\right)=0$, but also $u^{\prime}\left(r_{1}, \gamma\right)=0$, then $u(\cdot, \gamma)$ defines the (unique) solution $u$ of the free boundary problem (3.1), (3.3), where $R=r_{1}$.

If $r_{1}=\infty$, then $u(r, \gamma)$ has a positive minimum at some finite value of $r$, after which it oscillates with decreasing amplitude around the constant value 1.

Lemma 2 For $0<r<r_{1}$, we have $0<u(r, \gamma)<\gamma$.

Proof. The lemma is true for $0<r \leq r_{0}$ (cf. Lemma 1). Beyond $r_{0}$, we use a simple energy argument. The energy $E$ of any solution $u$ of (3.1), defined by the expression

$$
E(r)=\frac{1}{2}\left(u^{\prime}(r)\right)^{2}+\frac{1}{2}(u(r))^{2}-\frac{1}{q+1}(u(r))^{q+1},
$$

is a monotonically decreasing function of its argument, as $E^{\prime}(r)=-((2 \nu+1) / r)\left(u^{\prime}(r)\right)^{2} \leq 0$ for all $r \geq 0$.

Suppose the lemma were false for $r_{0}<r<r_{1}$. Then $u\left(r_{2}, \gamma\right)=\gamma$ for some $r_{2} \in\left(r_{0}, r_{1}\right)$, where $E\left(r_{2}\right) \geq \gamma^{2} / 2-\gamma^{q+1} /(q+1)=E(0)$, and we have a contradiction.

Let $w$ be defined in terms of $u(\cdot, \gamma)$ by the expression

$$
w(r)=\frac{r u(r, \gamma)}{\gamma} .
$$

This function is a solution of the initial value problem

$$
\begin{gathered}
w^{\prime \prime}+\frac{2 \nu-1}{r} w^{\prime}+\left(1-\frac{1}{(u(r, \gamma))^{1-q}}-\frac{2 \nu-1}{r^{2}}\right) w=0, r>0, \\
w(0)=0, w^{\prime}(0)=1 .
\end{gathered}
$$

It vanishes when $u$ vanishes, while its derivative vanishes when both $u$ and $u^{\prime}$ vanish. Furthermore,

$$
0<w(r)<r, 0<r<r_{1} .
$$

The following lemma gives a lower bound for $r_{1}$.

Lemma 3 We have $r_{1}>j_{\nu, 1} / \delta$, where

$$
\delta=\sqrt{1-\gamma^{-(1-q)}} .
$$


Proof. Because $u(r, \gamma)<\gamma, w$ oscillates less than the solution $v$ of the initial value problem

$$
v^{\prime \prime}+\frac{2 \nu-1}{r} w^{\prime}+\left(\delta^{2}-\frac{2 \nu-1}{r^{2}}\right) v=0, r>0 ; \quad v(0)=0, v^{\prime}(0)=1
$$

at least as long as $v$ is positive. Since $v(r)=2^{\nu} \Gamma(\nu+1) \delta^{-\nu} r^{1-\nu} J_{\nu}(\delta r)$, the first zero of $v$ occurs at $j_{\nu, 1} / \delta$. It must therefore be the case that $r_{1}>j_{\nu, 1} / \delta$.

\subsection{Bounds on $\left(0, j_{\nu, 1} / \delta\right)$.}

We rewrite the equation (3.9) in the form

$$
w^{\prime \prime}+\frac{2 \nu-1}{r} w^{\prime}+\left(\delta^{2}-\frac{2 \nu-1}{r^{2}}\right) w=f(w)
$$

where

$$
f(w)=\frac{1}{\gamma^{1-q}}\left\{\left(\frac{r}{w}\right)^{1-q}-1\right\} w
$$

Using the method of variation of parameters, we obtain the integral equation for $w$,

$$
w(r)=r g(\delta r)+\frac{\pi}{2} \int_{0}^{r} r^{1-\nu} s^{\nu}\left\{J_{\nu}(\delta s) Y_{\nu}(\delta r)-Y_{\nu}(\delta s) J_{\nu}(\delta r)\right\} f(w(s)) d s
$$

where

$$
g(\rho)=2^{\nu} \Gamma(\nu+1) \rho^{-\nu} J_{\nu}(\rho) .
$$

$J_{\nu}$ and $Y_{\nu}$ are the Bessel functions of the first and second kind, respectively, of order $\nu$. The expression (3.16) holds for all $r \in\left[0, r_{1}\right)$ or, if $r_{1}$ is finite, for all $r \in\left[0, r_{1}\right]$. We now restrict $r$ to the interval $\left[0, j_{\nu, 1} / \delta\right]$.

Lemma 4 For $0<r<j_{\nu, 1} / \delta$, we have

$$
0<r g(\delta r)<w(r)<r\left(g(\delta r)+\frac{\phi(\delta r)}{\log \gamma}\right)
$$

where $g$ is defined in (3.17) and

$$
\phi(\rho)=\frac{\rho^{2}(g(\rho))^{-1} \log (g(\rho))^{-1}}{4(\nu+1)} .
$$

Proof. Take any $r \in\left(0, j_{\nu, 1} / \delta\right)$. It follows from the Kneser-Sommerfeld expansion-see [2, Section 15.42]-that

$$
J_{\nu}(\delta s) Y_{\nu}(\delta r)-Y_{\nu}(\delta s) J_{\nu}(\delta r)=\frac{4 \delta r J_{\nu}(\delta r)}{\pi J_{\nu}(\delta s)} \sum_{n=1}^{\infty} \frac{\left(J_{\nu}\left(j_{\nu, n} s / r\right)\right)^{2}}{\left(j_{\nu, n}^{2}-(\delta r)^{2}\right) j_{\nu, n} J_{\nu}^{\prime 2}\left(j_{\nu, n}\right)}
$$


for $0 \leq s \leq r$. All the terms in the right member are positive, so the expression in the left member is positive. Furthermore, $f(w(s))$ is positive for $0 \leq s \leq r$. Therefore, the integral in (3.16) is positive. Obviously, $g(\delta r)$ is positive, so

$$
w(r)>r g(\delta r)>0,0<r<j_{\nu, 1} / \delta .
$$

From (3.21) and the fact that $g$ is decreasing on $\left(0, j_{\nu, 1}\right)$ we deduce that

$$
\frac{s}{w(s)}<\frac{1}{g(\delta s)} \leq \frac{1}{g(\delta r)}, 0 \leq s \leq r .
$$

Therefore,

$$
f(w(s))<\frac{(g(\delta r))^{-(1-q)}-1}{\gamma^{1-q}} w(s), 0 \leq s \leq r .
$$

Furthermore, $w(s) \leq s$, cf. (3.11). These inequalities lead to the estimate

$$
\begin{aligned}
& \frac{\pi}{2} \int_{0}^{r} r^{1-\nu} s^{\nu}\left\{J_{\nu}(\delta s) Y_{\nu}(\delta r)-Y_{\nu}(\delta s) J_{\nu}(\delta r)\right\} f(w(s)) d s \\
& \quad \leq r \frac{(g(\delta r))^{-(1-q)}-1}{\gamma^{1-q}-1}\left[\rho^{-\nu} \frac{\pi}{2} \int_{0}^{\rho}\left\{J_{\nu}(z) Y_{\nu}(\rho)-Y_{\nu}(z) J_{\nu}(\rho)\right\} z^{\nu+1} d z\right]_{\rho=\delta r} .
\end{aligned}
$$

The expression in square brackets can be evaluated by means of the recurrence formulae for Bessel functions-see [2, Section 3.2] - and the resulting expression can be simplified further by means of the Wronskian-see [2, Section 3.63]--,

$$
\rho^{-\nu} \frac{\pi}{2} \int_{0}^{\rho}\left\{J_{\nu}(z) Y_{\nu}(\rho)-Y_{\nu}(z) J_{\nu}(\rho)\right\} z^{\nu+1} d z=1-g(\rho) .
$$

We estimate this expression by substituting the series expansion for the Bessel function $J_{\nu}$ and truncating after the first term,

$$
1-g(\rho)=1-2^{\nu} \Gamma(\nu+1) \rho^{-\nu} J_{\nu}(\rho) \leq \frac{\rho^{2}}{4(\nu+1)} .
$$

To estimate the factor in front of the square brackets in $(3.24)$, we observe that $0<g(\delta r)<1$ on $\left(0, j_{\nu, 1} / \delta\right)$ and $\gamma>1$. Furthermore, one readily verifies that

$$
\frac{1-x^{1-q}}{\log x^{-1}} \leq \frac{y^{1-q}-1}{\log y}
$$

for any pair $(x, y)$ with $0<x \leq 1 \leq y$. Therefore,

$$
\begin{aligned}
& \frac{(g(\delta r))^{-(1-q)}-1}{\gamma^{1-q}-1}=(g(\delta r))^{-(1-q)} \frac{1-(g(\delta r))^{1-q}}{\gamma^{1-q}-1} \\
& \leq \frac{(g(\delta r))^{-(1-q)} \log (g(\delta r))^{-1}}{\log \gamma} \leq \frac{(g(\delta r))^{-1} \log (g(\delta r))^{-1}}{\log \gamma} .
\end{aligned}
$$

The lemma now follows from $(3.16),(3.21),(3.24),(3.25),(3.26)$, and (3.27). 
In terms of $u$, we have the following bounds:

$$
0<\gamma g(\delta r)<u(r, \gamma)<\gamma\left[g(\delta r)+\frac{\phi(\delta r)}{\log \gamma}\right], 0<r<\frac{j_{\nu, 1}}{\delta}
$$

Because $\phi(\rho)$ increases beyond bounds as $g(\rho)$ decreases to 0 , the upper bound in (3.18) or (3.28) increases indefinitely as $r$ approaches the right endpoint of the interval $\left(0, j_{\nu, 1} / \delta\right)$.

In the following analysis we also need an estimate of the quantity $r^{1-2 \nu}\left(r^{2 \nu-1} w\right)^{\prime}(r)$. It is given by the expression

$$
r^{1-2 \nu}\left(r^{2 \nu-1} w\right)^{\prime}(r)=h(\delta r)+\delta \frac{\pi}{2} \int_{0}^{r} r^{1-\nu} s^{\nu}\left\{J_{\nu}(\delta s) Y_{\nu-1}(\delta r)-Y_{\nu}(\delta s) J_{\nu-1}(\delta r)\right\} f(w(s)) d s,
$$

where

$$
h(\rho)=2^{\nu} \Gamma(\nu+1) \rho^{1-\nu} J_{\nu-1}(\rho) .
$$

Like (3.16), (3.29) holds for all $r \in\left[0, r_{1}\right)$ or, if $r_{1}$ is finite, for all $r \in\left[0, r_{1}\right]$. The following lemma gives an estimate on $\left(0, j_{\nu, 1} / \delta\right)$.

Lemma 5 For $0<r<j_{\nu, 1} / \delta$, we have

$$
\left|r^{1-2 \nu}\left(r^{2 \nu-1} w\right)^{\prime}(r)-h(\delta r)\right|<2(\nu+1) \frac{\phi(\delta r)}{\log \gamma},
$$

where $h$ is defined in (3.30) and $\phi$ is defined in (3.19).

Proof. The proof is similar to, although slightly more involved than the proof of Lemma 4. Instead of (3.16), we use (3.29). The analog of (3.24) is

$$
\begin{aligned}
& \delta \frac{\pi}{2} \int_{0}^{r} r^{1-\nu} s^{\nu}\left\{J_{\nu}(\delta s) Y_{\nu-1}(\delta r)-Y_{\nu}(\delta s) J_{\nu-1}(\delta r)\right\} f(w(s)) d s \\
& \quad \leq \frac{(g(\delta r))^{-(1-q)}-1}{\gamma^{1-q}-1}\left[\rho^{1-\nu} \frac{\pi}{2} \int_{0}^{\rho}\left\{J_{\nu}(z) Y_{\nu-1}(\rho)-Y_{\nu}(z) J_{\nu-1}(\rho)\right\} z^{\nu+1} d z\right]_{\rho=\delta r}
\end{aligned}
$$

The expression in square brackets can again be evaluated; instead of (3.25) we have

$$
\rho^{1-\nu} \frac{\pi}{2} \int_{0}^{\rho}\left\{J_{\nu}(z) Y_{\nu-1}(\rho)-Y_{\nu}(z) J_{\nu-1}(\rho)\right\} z^{\nu+1} d z=2(\nu+1)-h(\rho),
$$

where

$$
2(\nu+1)-h(\rho)=2(\nu+1)-2^{\nu} \Gamma(\nu+1) \rho^{1-\nu} J_{\nu-1}(\rho) \leq \frac{1}{2} \rho^{2} .
$$

The lemma follows from (3.29), (3.32), (3.33), (3.34), and (3.27). 


\subsection{Estimates at $r_{0}$.}

We use the results of Lemmas 4 and 5 to estimate $r_{0}$ and $r^{1-2 \nu}\left(r^{2 \nu-1} w\right)^{\prime}$ at $r_{0}$.

Lemma 6 Let $a \in\left(j_{\nu-1,1}, j_{\nu, 1}\right)$ be fixed. Then there exists a conrtant $\gamma_{1}>1$ that does not depend on $q$, such that

$$
r_{0}^{1-2 \nu}\left(r^{2 \nu-1} w\right)^{\prime}\left(r_{0}\right)<-\frac{1}{2}|h(a)|
$$

and

$$
\frac{a}{\delta}<r_{0}<\left(1+\frac{4 \nu}{|h(a)|}\right)^{1 /(2 \nu)} \frac{a}{\delta}
$$

for all $\gamma \geq \gamma_{1}$.

Proof. With the choice of $a$ indicated in the statement of the lemma we have $g(a)>0$ and $h(a)<0$. These inequalities follow from the interlacing property of the zeros of Bessel functions,

$$
0<j_{\nu, 1}<j_{\nu+1,1}<j_{\nu, 2}<j_{\nu+1,2}<j_{\nu, 3}<\ldots
$$

cf. [2, Section 15.22].

We begin by observing that $w$ oscillates less than $v$, where $v(r)=r g(\delta r), g$ defined by (3.17). Therefore $r_{0}$, which is defined by the identity $w(r)=r / \gamma$, is certainly beyond the point $r_{2}$, where $g\left(\delta r_{2}\right)=1 / \gamma$. Therefore, if

$$
\gamma_{0}=1 / g(a)
$$

then $g\left(\delta r_{2}\right) \leq g(a)$ for all $\gamma \geq \gamma_{0}$. Now, $g$ is monotonically decreasing between $a$ and $j_{\nu-1,1}$, so then we also have $\delta r_{2} \geq a$ for all $\gamma \geq \gamma_{0}$. Since $r_{0}>r_{2}$, we have thus achieved that

$$
a / \delta<r_{0}
$$

for all $\gamma \geq \gamma_{0}$.

With $r_{3}=a / \delta$, it follows from (3.31) that

$$
r_{3}^{1-2 \nu}\left(r^{2 \nu-1} w\right)^{\prime}\left(r_{3}\right)<-|h(a)|+2(\nu+1) \frac{\phi(a)}{\log \gamma} .
$$

Here, $h(a)$ and $\phi(a)$ do not depend on $q$ or $\gamma$. Therefore, if we now define $\gamma_{1}$,

$$
\gamma_{1}=\min \left\{\gamma_{0}, \exp \left(2(\nu+1) \frac{\phi(a)}{|h(a)|}\right)\right\}
$$

then $\gamma_{1}$ is independent of $q$ and

$$
r_{3}^{1-2 \nu}\left(r^{2 \nu-1} w\right)^{\prime}\left(r_{3}\right)<-\frac{1}{2}|h(a)|
$$

for all $\gamma \geq \gamma_{1}$. Writing the differential equation (3.9) in the form

$$
\left(r^{1-2 \nu}\left(r^{2 \nu-1} w\right)^{\prime}\right)^{\prime}=-\left(1-u^{-(1-q)}\right)
$$


we observe that the function $r^{1-2 \nu}\left(r^{2 \nu-1} w\right)^{\prime}$ is decreasing as long as $u(r, \gamma)>1$-that is, up to $r_{0}$. Therefore, the bound (3.41) extends to the entire interval $\left[r_{3}, r_{0}\right]$ and we have

$$
r^{1-2 \nu}\left(r^{2 \nu-1} w\right)^{\prime}(r)<-\frac{1}{2}|h(a)|, r_{3} \leq r \leq r_{0} .
$$

for all $\gamma \geq \gamma_{1}$. In particular, the inequality holds at $r_{0}$, as asserted in (3.35).

Multiplying both sides of the inequality (3.43) by $r^{2 \nu-1}$ and integrating over the interval $\left(r_{3}, r_{0}\right)$, we find

$$
\left(\frac{w\left(r_{3}\right)}{r_{3}}+\frac{|h(a)|}{4 \nu}\right) r_{3}^{2 \nu}-\frac{|h(a)|}{4 \nu} r_{0}^{2 \nu}>\frac{w\left(r_{0}\right)}{r_{0}} r_{0}^{2 \nu}
$$

Here, we estimate the expression in the right member from below by 0 . In the left member, we estimate the ratio $w\left(r_{3}\right) / r_{3}$ from above by 1 ; cf. (3.11). Thus,

$$
r_{0}^{2 \nu}<\left(1+\frac{4 \nu}{|h(a)|}\right) r_{3}^{2 \nu}
$$

The inequalities (3.36) now follow from (3.38) and (3.45).

\subsection{Down to 0.}

We are now in a position to prove that the continuation of $u$ beyond $r_{0}$ decreases to 0 for all sufficiently large $\gamma$, independently of $q$.

Lemma 7 There exists a constant $\gamma_{2}$ that does not depend on $q\left(\gamma_{2} \geq \gamma_{1}\right.$, where $\gamma_{1}$ is the constant introduced in Lemma 6), such that $r_{1}<\infty$ for all $\gamma \geq \gamma_{2}$.

Proof. The proof is by contradiction, where we assume that, for some $\gamma \geq \gamma_{1}$, the solution $u(\cdot, \gamma)$ of $(3.1),(3.2)$ is positive for all $r \geq 0$.

Consider the function $w$ defined by (3.8). By assumption, $w$ is positive for all $r>0$. Because $\left(r^{1-2 \nu}\left(r^{2 \nu-1} w\right)^{\prime}\right)^{\prime}=(r / \gamma)\left(u^{q}-u\right)$ and $u^{q}-u<1-q$ for $u>0$, we have

$$
\left(r^{1-2 \nu}\left(r^{2 \nu-1} w\right)^{\prime}\right)^{\prime}(r)<\frac{(1-q) r}{\gamma}, r>0 .
$$

Integrating (3.46) from $r_{0}$ to any point $r>r_{0}$, and using the estimate (3.35) at $r_{0}$, we find

$$
r^{1-2 \nu}\left(r^{2 \nu-1} w\right)^{\prime}(r)<-\frac{1}{2}|h(a)|+\frac{(1-q) r^{2}}{2 \gamma}, r>r_{0}
$$

for all $\gamma \geq \gamma_{1}$. Because $\gamma \delta^{2}=\gamma-\gamma^{q}>\gamma^{1-q}-1>(1-q) \log \gamma$, it follows that

$$
r^{1-2 \nu}\left(r^{2 \nu-1} w\right)^{\prime}(r)<-\frac{1}{2}|h(a)|+\frac{r^{2} \delta^{2}}{2 \log \gamma}, r>r_{0}
$$

for all $\gamma \geq \gamma_{1}$. 
Now we restrict $r$ to a compact interval $\left[r_{0}, r_{2}\right]$, where

$$
r_{2}=b / \delta \text {, }
$$

and $b>a$ is a suitably chosen constant. Defining the constant $\gamma_{2}$ by

$$
\gamma_{2}=\min \left\{\gamma_{1}, \mathrm{e}^{2 b^{2} /|h(a)|}\right\}
$$

we then have

$$
\frac{r^{2} \delta^{2}}{2 \log \gamma} \leq \frac{1}{4}|h(a)|, r_{0} \leq r \leq r_{2},
$$

for all $\gamma \geq \gamma_{2}$, so $(3.48)$ reduces to

$$
r^{1-2 \nu}\left(r^{2 \nu-1} w\right)^{\prime}(r)<-\frac{1}{4}|h(a)|, r_{0} \leq r \leq r_{2}
$$

for all $\gamma \geq \gamma_{2}$. Hence,

$$
w\left(r_{2}\right)<\left[(a / b)^{2 \nu} \frac{w\left(r_{0}\right)}{r_{0}}-\left(1-(a / b)^{2 \nu}\right) \frac{|h(a)|}{8 \nu}\right] r_{2} .
$$

Using (3.36) to estimate $w\left(r_{0}\right) / r_{0}$ and writing the inequality in terms of $u$, we thus find that

$$
u\left(r_{2}, \gamma\right)<(a / b)^{2 \nu}\left(1+\frac{4 \nu}{|h(a)|}\right)^{1 /(2 \nu)}-\gamma\left(1-(a / b)^{2 \nu}\right) \frac{|h(a)|}{8 \nu}
$$

for all $\gamma \geq \gamma_{2}$.

But now we have a contradiction, as the expression in the right member of this inequality certainly becomes negative for sufficiently large values of $\gamma$. We conclude therefore that $u(\cdot, \gamma)$ reaches the value 0 at some finite point $r_{1}$, as claimed.

\subsection{Completion of the Proof.}

According to Lemma $7, u(\cdot, \gamma)$ ceases to be positive at a finite point $r_{1}$ for all $\gamma \geq \gamma_{2}$, where $\gamma_{2}$ is a constant that does not depend on $q$. Obviously, $r_{1}$ depends on the value of $\gamma$; in fact, it decreases as $\gamma$ increases. Let

$$
\Gamma=\inf \left\{\gamma>1: r_{1}<\infty\right\} .
$$

If $\gamma=\Gamma$, then $u(\cdot, \gamma)$ reaches the $r$-axis with a horizontal slope, so $u(\cdot, \Gamma)$ defines the unique solution $u$ of the free boundary problem $(1.1){ }^{\prime}$ ?), where

$$
R=r_{1}(\Gamma) \text {. }
$$

As we observed at the beginning of Section 3.2, the function $u$ is monotone everywhere where it is positive. Hence, $\|u\|=\Gamma$. Obviously, $\Gamma$ depends on $q$. However, it follows from Lemma 7 that $1<\Gamma \leq \gamma_{2}$, so $\|u\|$ is bounded as $q \rightarrow 1(q<1)$.

It remains to investigate the behavior of $R$ as $q \rightarrow 1(q<1)$. Because $\Gamma$ is bounded, $\lim _{q \rightarrow 1} \Gamma^{1-q}=1$. Then it follows from (3.12) that $\lim _{q \rightarrow 1} \delta=0$ and therefore, by Lemma 3 , $\lim _{q \rightarrow 1} R=\infty$. Thus, the proof of the theorem is complete. 


\title{
3.7 Special Case: $N=3$.
}

In the special case $N=3\left(\nu=\frac{1}{2}\right)$, it is actually possible to find a lower bound for $R$ that shows that $R$ grows beyond bounds as $q \rightarrow 1$.

A simple energy argument gives the inequality

$$
0=E(R)<E(0)=\frac{\Gamma^{2}}{2}-\frac{\Gamma^{1+q}}{1+q}
$$

cf. (3.7). Hence,

$$
\Gamma^{1-q}>\frac{2}{1+q}
$$

Next, we use an energy argument for (3.9). If $\nu=\frac{1}{2}$, this equation reduces to

$$
w^{\prime \prime}+w-\Gamma^{-(1-q)} r^{1-q} w^{q}=0 \text {. }
$$

Hence,

$$
\left(w^{2}+w^{2}-\frac{2}{1+q} \Gamma^{-(1-q)} r^{1-q} w^{1+q}\right)^{\prime}=-2 \frac{1-q}{1+q} \Gamma^{-(1-q)} r^{-q} w^{1+q} .
$$

Upon integration over $(0, R)$, the left member yields -1 ; in the right member we use the inequality $w(r)<r$ to obtain the estimate

$$
\int_{0}^{R} r^{-q} w^{1+q} d r<\frac{1}{2} R^{2}
$$

Thus, using (3.58), we find that

$$
R>\sqrt{\frac{2}{1-q}} .
$$

\section{References}

[1] H. G. Kaper and M. K. Kwong, Free Boundary Problems for Emden-Fowler Equations, Differential and Integral Equations 3 (1990), 353--362.

[2] G. N. Watson, A Treatise on the Theory of Bessel Functions, 2nd ed. Cambridge University Press (1966).

\section{DISCLAIMER}

\begin{abstract}
This report was prepared as an account of work sponsored by an agency of the United States Government. Neither the United States Government nor any agency thereof, nor any of their employees, makes any warranty, express or implied, or assumes any legal liability or responsibility for the accuracy, completeness, or usefulness of any information, apparatus, product, or process disclosed, or represents that its use would not infringe privately owned rights. Reference herein to any specific commercial product, process, or service by trade name, trademark, manufacturer, or otherwise does not necessarily constitute or imply its endorsement, recommendation, or favoring by the United States Government or any agency thereof. The views and opinions of authors expressed herein do not necessarily state or reflect those of the United States Government or any agency thereof.
\end{abstract}



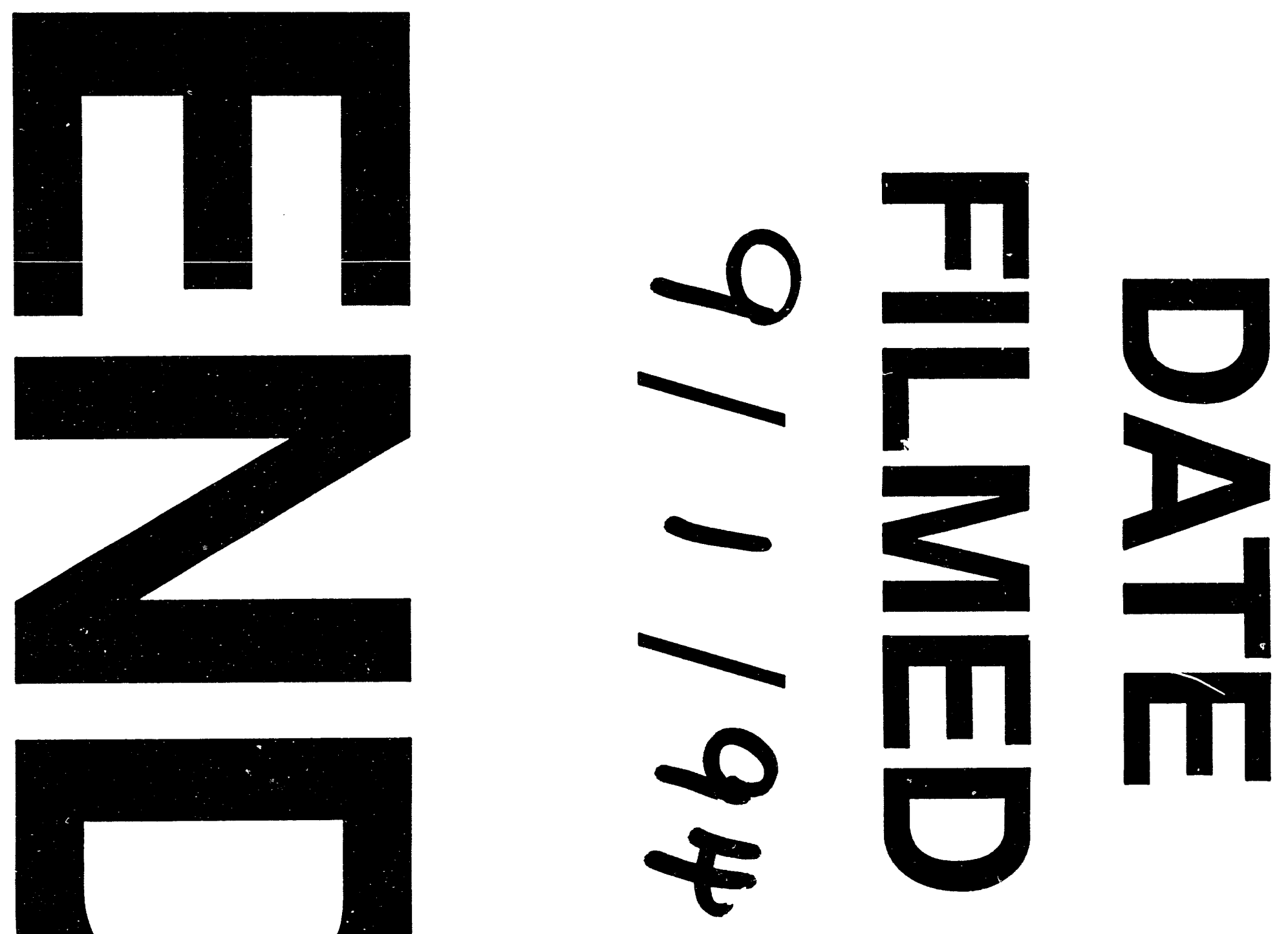
